Programming

Techniques and

Data Structures

Ian Munro

Editor

\title{
Planar Point Location Using Persistent Search Trees
}

\author{
NEIL SARNAK and ROBERT E. TARJAN
}

\begin{abstract}
A classical problem in computational geometry is the planar point location problem. This problem calls for preprocessing a polygonal subdivision of the plane defined by $n$ line segments so that, given a sequence of points, the polygon containing each point can be determined quickly on-line. Several ways of solving this problem in $O(\log n)$ query time and $O(n)$ space are known, but they are all rather complicated. We propose a simple O(log n)-query-time, $O(n)$-space solution, using persistent search trees. A persistent search tree differs from an ordinary search tree in that after an insertion or deletion, the old version of the tree can still be accessed. We develop a persistent form of binary search tree that supports insertions and deletions in the present and queries in the past. The time per query or update is $O(\log m)$, where $m$ is the total number of updates, and the space needed is $O(1)$ per update. Our planar point location algorithm is an immediate application of this data structure. The structure also provides an alternative to Chazelle's "hive graph" structure, which has a variety of applications in geometric retrieval.
\end{abstract}

\section{Planar poInt location}

Let us consider a classical geometric retrieval problem. Suppose the Euclidian plane is subdivided into polygons by $n$ line segments ${ }^{1}$ that intersect only at

\footnotetext{
${ }^{1}$ We regard a line or half-line as being a line segment, and an infinite region whose boundary consists of a finite number of line segments as being a polygon.
}

(C) 1986 ACM 0001-0782/86/0700-066975C their endpoints. (See Figure 1, p. 670.) Given such a polygonal subdivision and a sequence of query points in the plane, the planar point location problem is the problem of determining, for each query point, the polygon containing it. (For simplicity we shall assume that no query point lies on a line segment of the subdivision.) We require that the answers to the queries be produced on-line; that is, each query point must be located before the next one is knuwn.

A solution to the point location problem consists of an algorithm that preprocesses the polygonal subdivision, building a data structure that facilitates location of individual query points. We measure the efficiency of such a solution by three parameters: the preprocessing time, the space required to store the data structure, and the time per query. Of these, the preprocessing time is generally the least important.

Many solutions to the point location problem have been proposed [10,11, 13,18, 22, 23, 32]. If binary decisions are used to locate the query points, $\Omega(\log n)$ time per query is necessary. Dobkin and Lipton [11] showed that this lower bound is tight, exhibiting a method with $O(\log n)$ query time needing $O\left(n^{2}\right)$ space and preprocessing time. The Dobkin-Lipton result raised the question of whether an $O(\log n)$ bound on query time can be achieved using only $O(n)$ space, which is optimal if the planar subdivision must be stored. Liplon and Tarjan [23] answered this question affirmatively by devising a 
complicated method based on the planar separator theorem [24].

More recent research has focused on providing a simpler algorithm with resource bounds the same as or close to those of the Lipton-Tarjan method. Algorithms with $O(\log n)$ query time using $O(n)$ space have been developed by Kirkpatrick [18], who used the fact that every planar graph has an independent set containing a fixed fraction of the vertices; by Edelsbrunner, Guibas, and Stolfi [13], who improved a method of Lee and Preparata [22] that uses the notion of separating chains; and by Cole [10], who noted that the Dobkin-Lipton approach reduces planar point location to a problem of storing and accessing a set of similar lists.

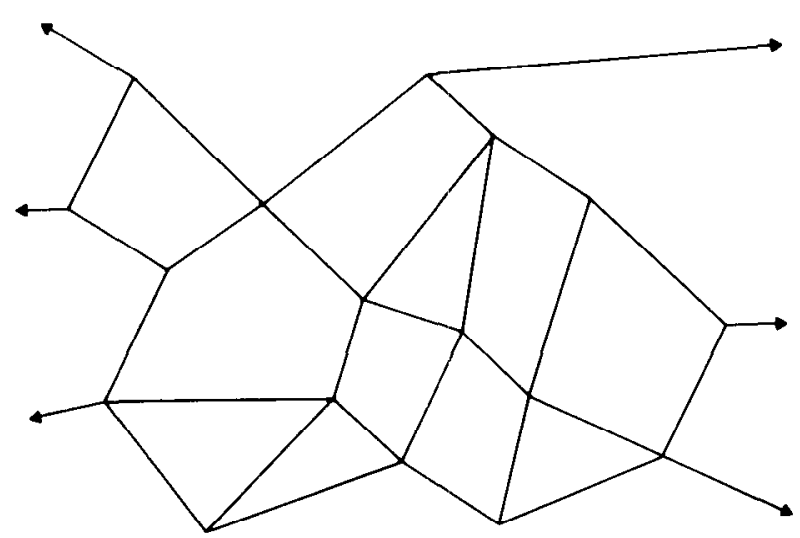

FIGURE 1. A Polygonal Subdivision. Arrows denote line segments going to infinity.

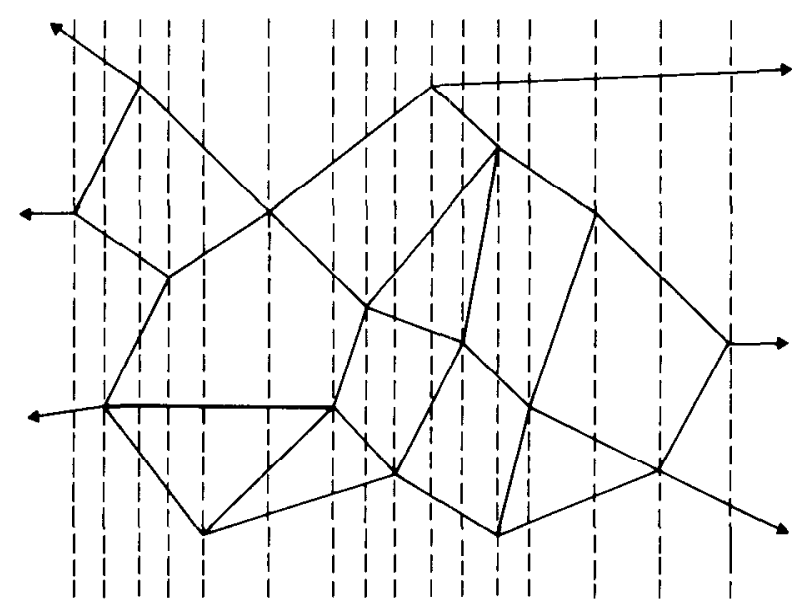

FIGURE 2. The Polygonal Subdivision of Figure 1 Divided into Slabs. The dashed lines are slab boundaries.
Cole's observation is the starting point for our work. Let us review the Dobkin-Lipton construction. Draw a vertical line through each vertex (intersection of line segments) in the planar subdivision. (See Figure 2.) This splits the plane into vertical slabs. The line segments of the subdivision intersecting a slab are totally ordered, from the bottom to the top of the slab. Associate with each line segment the polygon just above it. Now it is possible to locate a query point with two binary searches: the first, on the $x$-coordinate, locates the slab containing the point; the second, on the line segments intersecting the slab, locates the nearest line segment below the point, and hence determines the polygon containing the point. (By introducing a dummy line segment running from $(-\infty,-\infty)$ to $(\infty,-\infty)$, we can guarantee that below every point there is a line segment.) Since testing whether a point is above or helow a line segment takes $O(1)$ time, a point query takes $O(\log n)$ time. Unfortunately, if we build a separate search structure (such as a binary search tree) for each slab, the worst-case space requirement is $\Theta\left(n^{2}\right)$, since $\Theta(n)$ line segments can intersect $\Theta(n)$ slabs.

We can reduce the space bound by noticing as Cole did that the sets of line segments intersecting contiguous slabs are similar. Think of the $x$-coordinate as time. Consider how the set of line segments intersecting the current slab changes as the time increases from $-\infty$ to $+\infty$. As the boundary from one slab to the next is crossed, certain segments are deleted from the set and other segments are inserted. Over the entire time range, there are $2 n$ insertions and deletions, one insertion and one deletion per segment. ('Think of line segments going to $-\infty$ in the $x$-coordinate as being inserted at time $-\infty$, and line segments going to $+\infty$ in the $x$-coordinate as being deleted at time $+\infty$.)

We have thus reduced the point location problem to the problem of storing a sorted set subject to insertions and deletions so that all past versions of the set, as well as the current version, can be accessed efficiently. In general we shall call a data structure persistent if the current version of the structure can be modified and all versions of the structure, past and present, can be accessed. Ordinary data structures, which do not support access in the past, we call ephemeral.

Cole solved the point location problem by devising a persistent representation of sorted sets that occupies $O(m)$ space and has $O(\log m)$ access time, where $m$ is the total number of updates (insertions and deletions) starting from an empty set. However, his data structure has two drawbacks. First, his method is indirect, proceeding by way of an intermediate problem in which item substitutions but neither in- 
sertions nor deletions are alluwed. Second, the entire sequence of updates must be known in advance, making the data structure unusable in situations where the updates take place on-line. We shall propose a simpler data structure that overcomes these drawbacks.

Our main result, presented in Section 3, is a persistent form of binary search tree with an $O(\log m)$ worst-case access/insert/delete time and an amortized $^{2}$ space requirement of $O(1)$ per update. Our structure has neither of the drawbacks of Cole's. It provides a simple $O(n)$-space, $O(\log n)$ query-time point location algorithm. It can also replace Chazelle's "hive graph" [7], a rather complicated data structure with a variety of uses in geometric searching. Section 4 contains a brief discussion of these applications and some remarks about extensions and open problems. Some of the results presented here appear in preliminary form in Sarnak [34].

\section{PERSISTENT SORTED SETS AND SEARCH TREES}

We are now faced with a problem that is purely in the realm of data structures, the persistent sorted set problem. We wish to maintain a set of items that changes over time. The items have distinct keys, with the property that any collection of keys of items that are in the set simultaneously can be totally ordered. (The keys of two items that are not in the set at the same time need not be comparable.) Three operations on the set are allowed:

$\operatorname{access}(x, s, t)$ : Find and return the item in set $s$ at time $t$ with greatest key less than or equal to $x$. If there is no such item, return a special null item.

insert $(i, s, t$ ): At time $t$, insert item $i$ (with predefined key) into set $s$, assuming it is not already there. Item $i$ remains in the set until it is explicitly deleted.

delete $(i, s, t):$ At time $t$, delete item $i$ from set $s$, assuming it is there.

Starting with an empty set, we wish to perform on-line a sequence of operations, including $m$ updates (insertions and deletions), with the following property:

(*) Any update occurs at a time no earlier than any previous operation in the sequence. That is, updates are allowed only in the present.

The explicit time parameter $t$ in the operations formalizes the notion of persistence. We break ties in

${ }^{2}$ By amortized complexity we mean the complexity of an operation averaged over a worst-case sequence of operations. For a full discussion on this concept. see Tarjan's survey paper [39]. operation time by order in the sequence of operations. Property $\left({ }^{*}\right)$ allows accesses to take place either in the present (after the most recent update) or in the past. In the usual ephemeral version of the sorted set problem, the time of an operation is implicit, corresponding to its position in the sequence of operations. An equivalent definition of the ephemeral problem is obtained by requiring the sequence of operations to have the following stronger property in place of $\left({ }^{*}\right)$ : the operations in the sequence occur in nondecreasing order by time.

This problem and variants of it have been studied by many authors $[8,10,12,21,27,28,31,33,36]$. Dobkin and Munro [12] considered the problem of maintaining a persistent list subject to access, insertion, and deletion by list position. (The items in the list have positions 1 through $n$ counting from the front to the back of the list.) The persistent list problem seems to be harder than the persistent sorted set problem. Dobkin and Munro proposed an off-line method (all updates occur in the sequence before all accesses) with $O\left((\log m)^{2}\right)$ access time using $O(m \log$ $m$ ) space. Overmars [31] proposed an on-line method for the persistent list problem with $O(\log m)$ access time using $O(m \log m)$ space. Overmars also studied the much easier version of the persistent sorted set problem in which an operation $\operatorname{access}(x, t)$ need only return an item if the set contains an item with key exactly equal to $x$. For this version, he developed an $O(m)$-space, $O(\log m)$-access-time on-line algorithm. Chazelle [8] devised an $O(m)$-space, $O\left((\log m)^{2}\right)$ access-time method for the off-line version of the original persistent sorted set problem. As discussed in Section 1, Cole [10] discovered an $O(m)$-space, $O(\log m)$-access-time off-line algorithm.

All these methods use data structures that are somewhat ad hoc and baroque. A more direct approach is to start with an ephemeral data structure for sorted sets or lists and make it persistent. This idea was pursued independently by Myers [27, 28], Krijnen and Meertens [21], Reps, Teitelbaum, and Demers [33], and Swart [36], who independently proposed essentially the same idea, which we shall call path copying. The resulting data structure can be used to represent both persistent sorted sets and persistent lists with an $O(\log m)$ time bound рer operation and an $O(\log m)$ space bound per update.

In the remainder of this section we shall review binary search trees and how they can be made persistent using path copying. In Section 3 we propose a new method that uses space even more efficiently than path copying. It leads to a data structure for persistent sorted sets (but not persistent lists) that has bounds of $O(\log m)$ worst-case time per operation and $O(1)$ amortized space per update. 


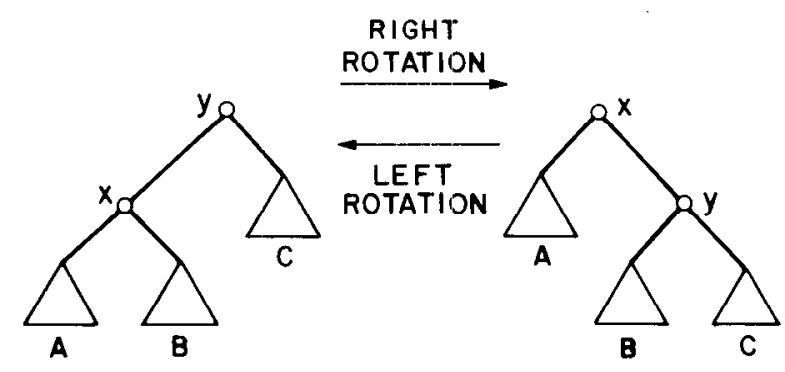

FIGURE 3. A Rotation in a Binary Tree. The tree can be a subtree of a larger tree.

A standard data structure for representing ephemeral sorted sets is the binary search tree. This is a binary tree ${ }^{3}$ containing the items of the set in its nodes, one item per node, with the items arranged in symmetric order: if $x$ is any node, the key of the item in $x$ is greater than the keys of all items in its left subtree and less than the keys of all items in its right subtree. The symmetric-order item arrangement allows us to perform an access operation by starting at the tree root and searching down through the tree, along a path determined by comparisons of the query key with the keys of items in the tree: if the query key is equal to the key of the item in the current node, we terminate the access by returning the item in the current node; if it is less, we proceed to the left child of the current node; if it is greater, we proceed to the right child. Either the search terminates having found an item with key equal to the query key, or it runs off the bottom of the tree. In the latter case, we return the item in the node from which the search last went right; if there is no such node, we return null.

The time for an access operation in the worst case is proportional to the depth of the tree. If the tree is binary, its depth is at least $\lfloor\log n\rfloor+1$, where $n$ is the number of tree nodes. This bound is tight for balanced binary trees, which have depth $O(\log n)$ and insertion and deletion time bounds of $O(\log n)$ as well. There are many types of balanced trees, including AVL or height-balanced trees [1], trees of bounded balance or weight-balanced trees [29], and redblack trees [14]. In such trees balance is maintained by storing certain balance information in each node (of a kind that depends upon the type of tree) and rebalancing after an insertion or deletion by performing a series of rotations along the access path (the path from the root to the inserted or deleted item). A rotation (see Figure 3) is a local transformation that changes the depths of certain nodes, pre-

${ }^{3}$ See the books of Knuth [19] and Tarjan [37] for our tree terminology. serves symmetric order, and lakes $O(1)$ lime, assuming that a standard binary tree representation is used such as storing two pointers in each node, to its left and right children.

For definiteness, we shall concentrate on redblack trees, although our ideas apply to certain other kinds of balanced trees. In a red-black tree each node has a color, either red or black, subject to the following constraints:

(i) all missing (external) nodes are regarded as black;

(ii) all paths from the root to a missing node contain the same number of black nodes;

(iii) any red node, if it has a parent, has a black parent.

This definition is due to Guibas and Sedgewick [14]. Bayer [3] introduced these trees, calling them symmetric binary B-trees. Olivie [30] gave an equivalent definition (see [38]) and used the term halfbalanced trees.

Updating red-black trees is especially efficient as compared to updating other kinds of balanced trees. Rebalancing after an insertion or deletion can be (a)

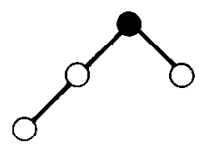

(b)<smiles></smiles>

(c)

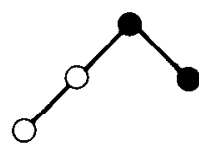

(d)

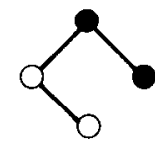

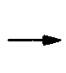

OR
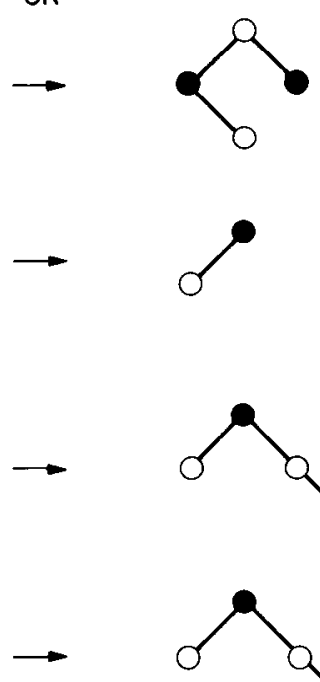
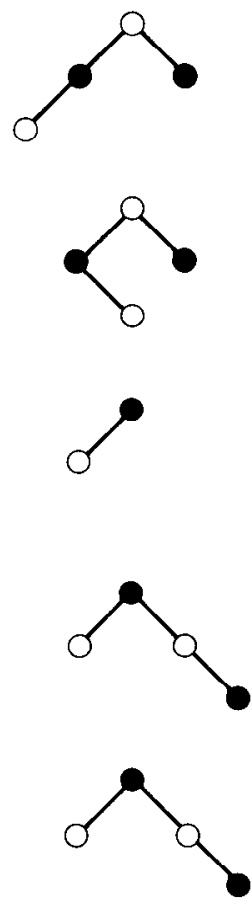

FIGURE 4. The Rebalancing Transformations in Red-Black Tree Insertion. Symmetric cases are omitted. Solid nodes are black; hollow nodes are red. All unshown children of red nodes are black. In cases (c) and (d) the bottommost black node can be missing. 
done in $O(1)$ rotations and $O(\log n)$ color changes [38]. Furthermore the number of color changes per update is $O(1)$ in the amortized case $[15,16,25]$. Rebalancing is a bottom-up process. To perform an insertion, we proceed as in an access operation. At the place where the search runs off the bottom of the tree, we attach a new node containing the new item. We color this node red. This preserves the black constraint (ii) but may violate the red constraint (iii). If there are now two red nodes in a row the topmost of which has a red sibling, we color the topmost red node, its red sibling black, and their common parent (which must be black) red. (See Figure 4a.) This may produce a new violation of the red constraint. We repeat the transformation of Figure $4 \mathrm{a}$, moving the violation up the tree, until this transformation no longer applies. If there is still a violation, we apply the appropriate transformation among those in Figure 4b, c, and d to eliminate the violation. This terminates the insertion. The only rotations are in the terminal cases: Figure $4 \mathrm{c}$ takes one rotation and Figure 4d takes two.

A deletion is similar. We first search for the item to be deleted. If it is in a node with a left child, we swap the item with its predecessor (in symmetric order), which we find by taking a left branch and then right branches until reaching a node with no right child. Now the item to be deleted is in a node with at most one child. We delete this node and replace it with its child (if any). This does not affect the red constraint but will violate the black constraint if the deleted node was black. If there is a violation, the replacing node (which may be missing) is short; paths down from it contain one fewer black node than paths down from its sibling. We bubble the shortness up the trec by repeating the recoloring transformation of Figure 5a until it no longer applies. Then we perform the transformation of Figure $5 \mathrm{~b}$ if it applies, followed if necessary by one application of Figure $5 \mathrm{c}, \mathrm{d}$, or e. The maximum number of rotations needed is three.

Let us now consider how to make red-black trees persistent. We need a way to retain the old version of the tree when a new version is created by an update. We can of course copy the entire tree each time an update occurs, but this takes $O(n)$ time and space per update. The idea of Myers [27, 28], Krijnen and Meertens [21], Reps, Teitelbaum, and Demers [33], and Swart [36] is to copy only the nodes in which changes are made. Any node that contains a pointer to a node that is copied must itself be copied. Assuming that every node contains pointers only to its children, this means that copying one node requires copying the entire path to the node from the root of the tree. Thus we shall call this method path copying. The effect of this method is to create a set of search trees, one per update, having different roots but sharing common subtrees. Since node colors are needed only for update operations, all of which take place in the most recent version of the tree, we need not copy a node when its color changes; we merely overwrite the old color. This saves a constant factor in space. (See Figure 6, p. 674.)

The time and space per insertion or deletion in a persistent red-black tree is $O(\log n)$ since such an operation changes only nodes along a single path in the tree. If the update times are arbitrary real numbers, we must build an auxiliary structure to facilitate access to the appropriate root when searching in the past. An array of pointers to the roots, ordered by time of creation, suffices. We can use binary search in this array to access the appropriate root. This increases the time per access from $O(\log n)$ to $O(\log m)$. If we use exponential search, the time to perform an access in the $t$ th version of the tree can be reduced to $O(\log n+\log t)$ : we examine the first, second, fourth, ..., $2\lceil\log t\rceil$ th root until finding one created after the desired search time; then we use

(a)

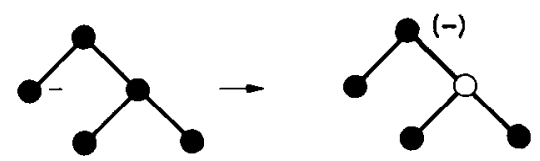

(b)

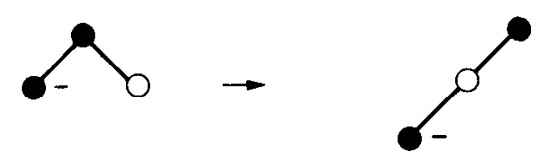

(c)

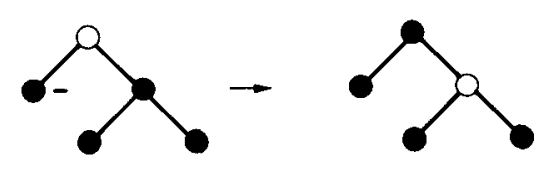

(d)

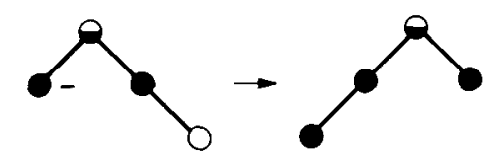

(e)
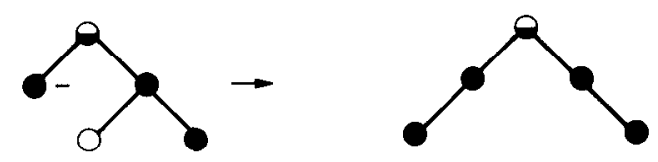

FIGURE 5. The Rebalancing Transformation in Red-Black Tree Deletion. The two ambiguous (half-solid) nodes in (d) have the same color, as do the two in (e). Minus signs denote short nodes. In (a), the top node after the transformation is short unless it is the root. 


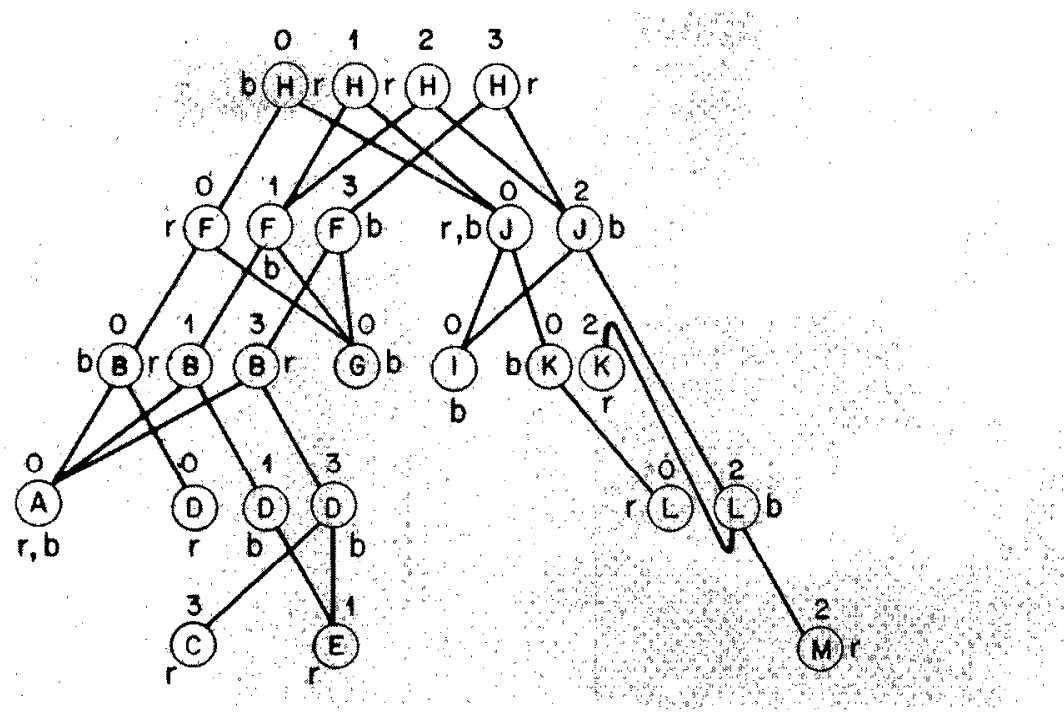

FGURE 6. A Persistent Red-Black Tree With Path Copying. The initial tree, existing at time O, contains A, B, D, F, G, H, I, J, K. Item E is inserted at time 1 , item $M$ at time 2 , and ltem $C$ at time 3 . The nodes are labeled by their colors; $\boldsymbol{f}$ fot red, $b$ for black. The nodes are also labeled by their time of creation. All edges exit the bottoms of nodes and enter the tops.

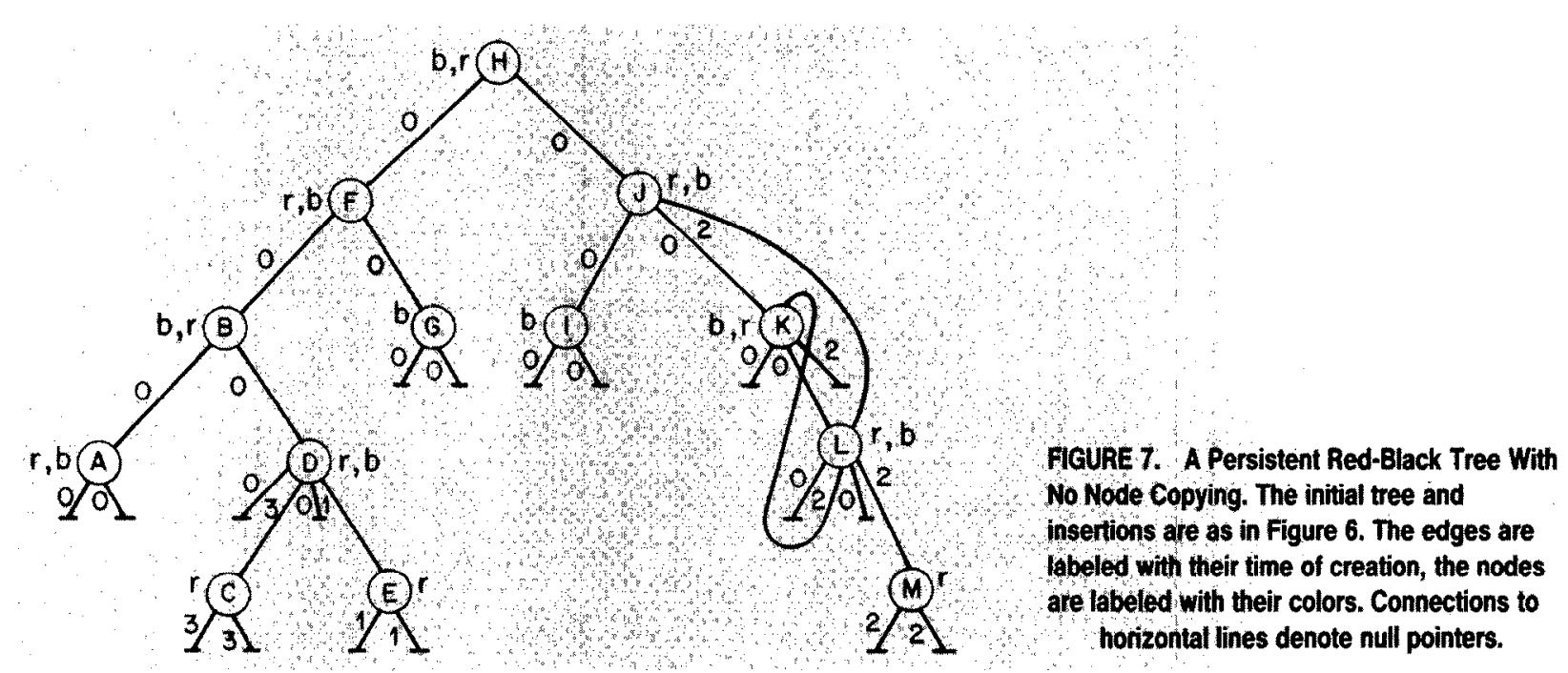

binary search on the roots from 1 through $2\lceil\log t\rceil$ (numbered in creation order). The same kind of search starting from the most recently created root and proceeding to earlier roots gives an access time of $O(\log n+\log (m-t))$. If the update times are the integers 1 through $m$, we can use direct access into the root array to provide $O(1)$-time access to the appropriate root, and the total time for an access operation is only $O(\log n)$.

As Swart noted, path copying works on any kind of balanced tree, not just on red-black trees. Myers used AVL trees, Krijnen and Meertens used B-trees, and Reps, Teitelbaum, and Demers used 2,3 trees. Path copying is also quite versatile in the applications it supports. By storing in each node the size of the subtree rooted there, we can obtain an imple- mentation of persistent lists (in which access is by list position rather than by key). We also have the ability to update any version, rather than just the current one, provided that an update is assumed to create an entirely new version, independent of all other versions. In order to have this more general kind of updating, we must copy a node when its balance information changes as well as when one of its pointers changes, but this increases the time and space needed for updates by only a constant factor.

\section{SPACE-EFFICIENT PERSISTENT SEARCH TREES}

A major drawback of the path copying method is its nonlinear space usage. In this section we shall propose a method that needs only linear space. We shall 
use the fact that old balance information need not be saved, although this is not essential. Our approach is to avoid copying the entire access path each time an update occurs. That this approach might work is suggested by the observation that in an ephemeral red-black tree, only $O(1)$ pointer changes are needed per update.

Suppose we implement persistent red-black trees without any node copying, by allowing nodes to become arbitrarily "fat": each time we want to change a pointer, we store the new pointer in the node, along with a time stamp indicating when the change occurred and a bit that indicates whether the new pointer is a left or right pointer. (This bit is actually redundant, since we can determine whether a pointer is left or right by comparing the key of the item in the node containing the pointer to that of the item in the node indicated by the pointer.) When a node color is changed we overwrite the old color. (See Figure 7.)

With this approach an insertion or deletion in a persistent red-black tree takes only $O(1)$ space, since an insertion creates only one new node and either kind of update causes only $O(1)$ pointer changes. The drawback of the method is its time penalty: since a node can contain an arbitrary number of left or right pointers, deciding which one to follow during a search is not a constant-time operation. If we use binary search by time stamp to decide which pointer to follow, choosing the correct pointer takes $O(\log m)$ time, and the time for an access, insertion, or deletion is $O((\log n)(\log m))$.

We can eliminate this time penalty by introducing limited node copying. We allow each node to hold $k$ pointers in addition to its original two. We choose $k$ to be a small positive constant; $k=1$ will do. When attempting to add a pointer to a node, if there is no empty slot for a new pointer, we copy the node, setting the initial left and right pointers of the copy to their latest values. (Thus the new node has $k$ empty slots.) We must also store a pointer to the copy in the latest parent of the copied node. If the parent has no free slot, it, too, is copied. Thus copying proliferates through successive ancestors until the root is copied or a node with a free slot is reached. (See Figure 8.)

Searching the resulting data structure is quite easy: when arriving at a node, we determine which pointer to follow by examining the key to decide whether to branch left or right and examining the time stamps of the extra pointers to select among multiple left or multiple right pointers. (We follow the pointer with the latest time stamp no greater than the search time if there is one, or else the initial pointer.) As noted in Section 2, if the update times are arbitrary real numbers we must build an auxiliary array to guide access operations to the proper roots. This makes the time for an access operation $O(\log m)$, whereas the time for an update operation is $O(\log n)$. However, in practice the number of roots is likely to be much smaller than $m$, since a root will be duplicated relatively infrequently. If the update times are consecutive integers, the auxiliary array provides $O(1)$-time access to the roots.

It remains for us to analyze the space used by the data structure. As with path copying, a single update operation using limited node copying can result in $O(\log n)$ new nodes. However, amortized over a sequence of updates, there are only $O(1)$ nodes copied per update, implying an $O(n)$ space bound for the

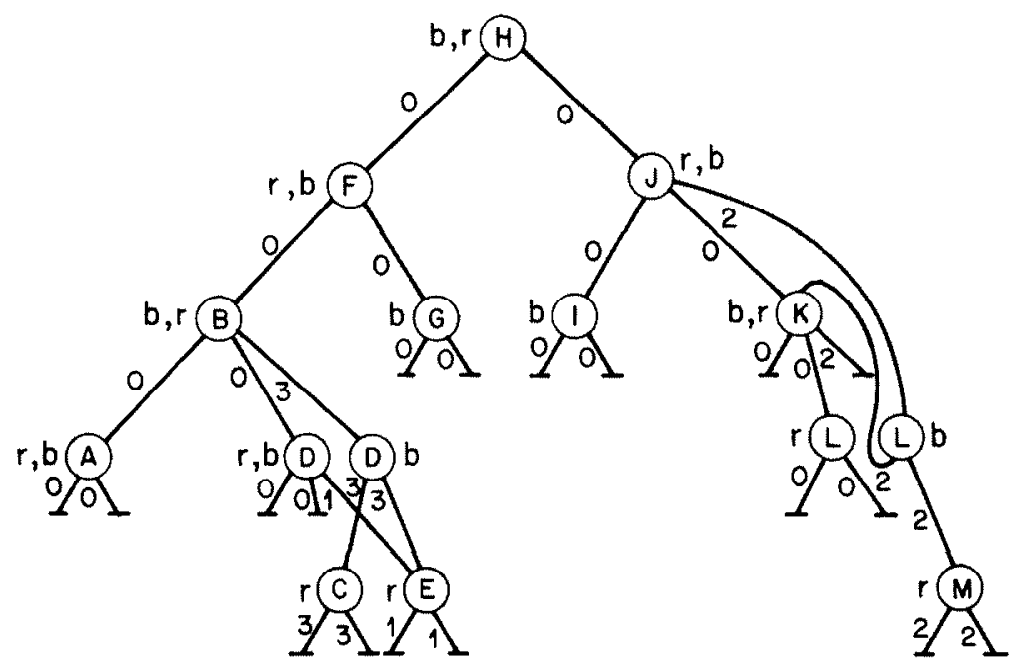

FIGURE 8. A Persistent Red-Black Tree With Limited Node Copying Assuming Each Node Can Hold One Extra Pointer. The initial tree and insertions are as in Figure 6. The labeling is as in Figure 7 . 
data structure. To obtain the amortized space bound we need some definitions. We partition the nodes of the data structure into two classes, live and dead. The live nodes are those reachable from the latest tree root by following pointers valid at the current time (the time of the most recent update). The live nodes form the current version of the search tree. As the current time increases, the node partition changes: live nodes can become dead but not viceversa. All nodes dead at a given time are not affected by any later update.

Our analysis uses the potential paradigm [39]. We define the potential of the data structure to be the number of live nodes minus $1 / k$ times the number of free slots in live nodes. We define the amortized space cost of an update operation to be the actual number of nodes it creates plus the net increase in potential it causes. With these definitions, the actual number of nodes created by a sequence of updates is bounded by the sum over all updates of the amoritzed space cost plus the net decrease in potential over the sequence. If we start with an empty data structure, the inilial potential is zero, and since the potential is always nonnegative the total amortized space cost is an upper bound on the actual number of nodes created.

The definition of potential is such that copying a node has an amortized space cost of zero, since a live node with no free slots becomes dead and a new live node with $k$ free slots is created, for a net decrease in potential of one, balancing the one new node created. Storing a new pointer in a node has an amortized space cost of $1 / k$. The creation of a new node during an insertion has an amortized space cost of one. Since an insertion or deletion requires storing $O(1)$ new pointers not counting node copying, the amortized space cost of an update is $O(1)$. A more careful count shows that an insertion has an amortized space cost of at most $1+6 / k$; a deletion, at most $7 / k$. In the special case of $k=1$, the amortized space cost per update is slightly less than indicated by these bounds: at most six for an insertion or deletion.

The choice $k=1$ is probably the most convenient in practice and is certainly the easiest to implement. However, choosing a larger value of $k$ may reduce the space needed hy the data structure, since although the space per node increases, the number of node copyings decreases. The best choice of $k$ depends on the exact way nodes are stored in memory and on the average (as opposed to worst-case) number of new pointers created by updates. Nevertheless, we shall give a simplified analysis based on the amorlized bounds derived above. Suppose that memory is divided into words, each of which is large enough to hold an item, a time stamp, or a pointer. We shall ignore the space needed to store node colors and the types of extra pointers (left or right); as noted above the latter information is redundant and the color of a node can if necessary be encoded by swapping or not swapping the original left and right pointers in a node. Under these assumptions a node requires $2 k+3$ words of memory, and the amortized space cost in words per update is at most $(2 k+3)(1+6 / k)=2 k+18 / k+15$. This is minimized at 27 words per update for $k=3$. This choice is only marginally better than the 30 words per update (six nodes of five words each) needed for $k=1$. Both these estimates are probably much larger than the expected values.

Limited node copying applied to red-black trees provides a linear-space representation of persistent sorted sets but not of persistent lists, because to represent lists we must maintain subtree sizes for all versions, and each update causes $O(\log n)$ subtree sizes to change. Limited node copying becomes similar to path copying in this case, and the space bound per update is $O(\log n)$. Our data structure does, however, support operations on persistent sorted sets in addition to those defined in Section 2. In particular, the following three operations are easy to handle:

access range $(x, y, s, t)$ : Find and return all items in set $s$ at time $t$ with key between $x$ and $y$ (inclusive).

join $\left(s_{1}, s_{2}, t\right):$ At time $t$, combine sets $s_{1}$ and $s_{2}$ into a single set, named $s_{1}$. Set $s_{2}$ becomes empty at time $t$. This operation requires that at time $t$ all items in $s_{1}$ have keys less than those of all items in $s_{2}$. Time $t$ can be any time greater than or equal to the time of the most recent update.

split $\left(s_{1}, s_{2}, x, t\right):$ At time $t$, split $s_{1}$ into two sets: a new version of $s_{1}$, containing all items with key less than or equal to $x$, and $s_{2}$, containing all items with key greater than $x$. Time $t$ can be any time greater than or equal to the time of the most recent update.

We shall discuss how to implement these operations on ephemeral red-black trees; the extensions to persistent trees are straightforward. To perform access range $(x, y, s, t)$, we proceed as in access $(x, s, t)$, thereby locating the node $e$ containing the item with smallest key no less than $x$. Then we visit the tree nodes starting from $e$ in symmetric order, stopping when we reach one containing an item with key exceeding $y$. In an ephemeral tree, the time for such a query is $O(k+\log n)$, where $k$ is the number of 
items returned. In a persistent tree the time is $O(k+\log m)$ assuming update times are arbitrary real numbers.

To discuss joining and splitting, we need the concept of the rank $r(e)$ of a node $e$, defined to be the number of black nodes on any path from $e$ down to a missing node. We can compute the rank of a node in time proportional to the rank by walking down from the node along any path. (Instead of comparing ranks from scratch, we can store with each tree root its rank, and then compute ranks on the way down the tree along any search path.) Consider a join of sets $s_{1}$ and $s_{2}$. To perform the join, we delete the item, say $i$, of smallest key in set $s_{2}$. We compute the ranks $r_{1}$ and $r_{2}$ of the roots of the trees $T_{1}$ and $T_{2}$ representing $s_{1}$ and the new $s_{2}$, respectively. Assume $r_{1} \geqslant r_{2}$. (The case $r_{1} \leqslant r_{2}$ is symmetric.) If $r_{1}=r_{2}$, we create a new black node containing $i$ and make the roots of $T_{1}$ and $T_{2}$ its children. If $r_{1}=r_{2}+1$ and the root of $T_{2}$ is red, we color it black and proceed as in the case of $r_{1}=$ $r_{2}$. Otherwise, we color the root of $T_{2}$ black if it is red and locate the node $e$ along the right path ${ }^{4}$ of $T_{1}$ of one higher rank than the root of $T_{2}$. We create a new red node containing $i$, which becomes the new right child of $\varepsilon$; its left child is the old right child of $e$ and its right child is the root of $T_{2}$. This may create a violation of the red constraint, which we eliminate as in insertion. The total time taken by the join is $O(\log n)$, where $n$ is the size of the new tree. Since only one new node is created and $O(1)$ pointer changes are made, the amortized space bound in the persistent version is $O(1)$. Note that once $i$ is deleted from $T_{2}$, and $r_{1}$ and $r_{2}$ are computed, the time for the rest of the join is $O\left(r_{1}-r_{2}+1\right)$. Furthermore, the rank of the root of the new tree is either $r_{1}$ or $r_{1}+1$.

We implement splitting using repeated joining. The easiest way to describe the algorithm is recursively. Suppose we have a procedure join 3 whose effect is as follows:

join3 $(e, f, g)$ : Let $e, f, g$ be nodes such that $e$ and $g$ are the roots of red-black trees $T_{1}$ and $T_{2}$, respectively, satisfying the condition that all items in $T_{1}$ have keys smaller than that of the item in $f$ and all items in $T_{2}$ have keys greater than that of the item in $f$. Combine $T_{1}, f$, and $T_{2}$ into a single tree whose root has rank max $\{r(e), r(g)\}$ or $\max \{r(e)$, $r(g)\}+1$, and return the root of the new tree.

We implement join3 in the same way as the second half of a binary join; the time it requires is $O(|r(e)-r(g)+1|)$. Using join3, we can implement a procedure split $(e, x)$, whose input is the root $e$ of a

4 The right path of a hinary tree is the path from the ront through right children to a missing node. The left path is defined similarly. red-black $T$ and a key $x$, and whose output is a pair $(f, g)$ such that $f$ and $g$ are the roots of the trees formed when $T$ is split at $x$. Let left(e) and right(e) be the left and right children of node $e$, respectively. To perform split $(e, x)$, we test whether the key of the item in $e$ is less than, or equal to $x$. If so, we perform split(right(e), $x)$, returning $(h, g)$. Then we compute $f=$ join3 (left $(e), e, h)$ and return $(f, g)$. The case of $x$ less than the key of the item in $e$ is symmetric.

The splitting algorithm has a running time of $O(\log n)$, because the multiple joins that take place have running times that form a telescoping sum, summing to $O(\log n)$. (See, for example [2].) The persistent version has an amortized space bound of $O(\log n)$. We can reduce this amortized space bound to $O(\log \min \{k, n-k\})$, where $k$ and $n-k$ are the sizes of the trees resulting from the split, by modifying the splitting algorithm slightly. To split a tree $T$ with root $r$ at key $x$, we follow the search path for $x$ until it changes direction. Suppose the first change of direction is from right to left (the opposite case is symmetric), and let $e$ be the node from which we branch left. (Node $e$ is the last node along the search path that is on the right path of $T$.) We break the link connecting $e$ to its parent $f$ and perform split $(e$, $x$ ) (as implemented above) returning $(g, h)$. We replace $f$ as the right child of its parent by left $(f)$, repairing the possible violation of the color constraints as in the deletion algorithm. Finally, we return the pair (join $3(r, f, g), h$ ). The time bound is still $O(\log n)$. The amortized space bound of $O(\log \min \{k$, $n-k$ ) for the persistent version follows from two facts: (i) node $e$ has rank $O(\log \min \{k, n-k\})$ in the original tree; (ii) restoring the color constraints after replacing node $f$ by its left child takes only $O(1)$ pointer changes.

Maintaining more than one persistent sorted set (as one must do if joins and splits are allowed) requires the maintenance of an auxiliary structure for each set to facilitate access to the appropriate root when searching. If multiple arrays are hard to use as auxiliary structures because of the problem of allocating storage for them, search trees can be used instead. The trees can be either ordinary balanced trees or some other kind, such as finger search trees ${ }^{5}$ or self-adjusting trees [35]. Depending on the choice of structure, the time to access the appropriate root is $O(\log m)$ or faster.

We conclude this section with a few remarks about the generality of our $O(1)$ amortized space bound for insertion, deletion, and join. What makes the analysis work is that red-black trees need only

${ }^{5}$ A finger search tree is a search tree augmented with a few pointers to favored nodes, called fingers. Access and update operations in the virinity of fingers are especially efficient $[6,16,17,20,41]$. 
O(1) pointer changes per update. This bound happens to be worst-case, but for our purpose an amorlized bound would do as well, since the resulting space bound is amortized anyway. This means that any kind of balanced tree with $O(1)$ amortized structural update times can be used in place of red-black trees. Examples include red-black trees with topdown instead of bottom-up updating [26], weightbalanced trees [5], and "weak" or "hysterical" $B$-trees $[15,16,25]$. We also have the option of storing the items in the external nodes of the tree instead of in the internal nodes (if we store appropriate keys in the internal nodes to guide searches).

\section{APPLICATIONS AND EXTENSIONS}

We have proposed a data structure for representing persistent sorted sets. Our structure has $O(\log m)$ access time, $O(\log n)$ update time, and needs $O(1)$ amortized space per update starting from an empty set. Here $n$ is the current set size and $m$ is the total number of updates. Our resource bounds match those of Cole [10], but our data structure is on-line and is simple enough to have potential practical applications.

As discussed in Section 1, our structure provides an efficient solution to the planar point location problem. For a planar subdivision of $n$ line segments, the preprocessing time necessary to build the data structure is $O(n \log n)$, the space needed is $O(n)$, and the query time is $O(\log n)$. Although these bounds have been obtained by others $[10,14,18,23]$, our method is simple enough to be useful in practice as well as efficient in theory. The methods of Kirkpatrick [18] and Edelsbrunner, Guibas, and Stolfi [13], when combined with a new linear-time algorithm for triangulating simple polygons [40], need $O(n)$ preprocessing time rather than $O(n \log n)$. Whether this reduction is important depends on the application. It is open whether some variant of our method has $O(n)$ preprocessing time.

Our structure also supports a generalization of the planar point location problem in which the queries are of the following form: given a vertical line segment, report all polygons the segment intersects. Such a query is equivalent to an access range operation on the corresponding persistent sorted set and thus takes $O(\log n+k)$ time where $k$ is the number of reported polygons. This bound has also been obtained by Chazelle [7], but only by using a complicated data structure, the hive graph, which is built as an extension to a data structure for the planar point location problem. Our structure solves both problems at once.

Chazelle gives a number of applications of hive graphs to geometric retrieval problems; for each of these, our structure provides a simpler solution. As an example, given a collection of line segments in the plane with $i$ crossings, we can in $O((n+i) \log n)$ time construct a data structure of size $O(n+i)$ that, given a vertical query segment, will allow us to report all data line segments the query segment crosses in $O(\log n+k)$ time, where $k$ is the number of reported segments. Cole [18] gives several other applications to which our structure applies.

We have obtained several extensions to the result presented here, which we shall discuss in detail in a future paper. The limited node copying technique generalizes to show that any ephemeral linked data structure, provided its nodes have constant indegree as well as constant out-degree, can be made persistent at an amortized space cost of $O(1)$ per structural change and an additive $O(\log m)$ time penalty per access. Whereas limited node copying as discussed in the present paper resembles nodesplitting in B-trees, the generalized technique resembles the "fractional cascading" idea of Chazelle and Guibas [9]. Among other applications, the generalized technique allows the addition of extra pointers, such as parent pointers and level links [6], to persistent red-black trees.

Our implementation of persistent search trees, although more space-efficient than the path copying method, is not as versatile. For example, path copying provides a representation for persistent lists as well as persistent sorted sets. For the list application, limited node copying is equivalent to path copying because the size information necessary for access by position must be updated all the way along an access path after any insertion or deletion, causing $\Theta(\log n)$ space usage per update. As noted in Section 2, path copying also provides the ability to update any version, rather than just the current one. Adding additional pointers, such as parent pointers, to the resulting data structure seems difficult. Nevertheless, path copying can be extended to finger search trees, reducing the space usage for updates in the vicinity of fingers.

There are many open problems concerning geometric retrieval problems and persistent data structures. Perhaps one of the most interesting is how to make our planar point location algorithm, or any such algorithm, dynamic, so that line segments can bo inserted and deleted on linc. The dynamization techniques of Bentley and Saxe [4] provide a way to handle insertions while preserving the $O(1)$ space bound. However, the access and insertion time becomes $\left.O(\log n)^{2}\right)$. Deletion seems to be harder to handle. An even more challenging problem is to find a persistent representation for a dynamically changing planar subdivision. A good data structure for this purpose would have many applications in computational geometry [10]. 


\section{REFERENCFS}

1. Adelson-Vels̀kii, G.M., and Landis, E.M. An algorithm for the organization of information. Sovief Math. Dokl. 3, 5 (Sept. 1962) $1259-1262$.

2. Aho, A.V., Hopcroft, J.E.. and Ullman, J.D. The Design and Analysis of Computer Algorithms. Addison-Wesley, Reading, Mass., 1974.

3. Bayer, R. Symmetric binary B-trees: Data structure and maintenance algorithms. Acta Inform. 1. 4 (Nov. 1972), 290-306.

4. Bentley, J.L., and Saxe, J.B. Decomposable searching problems I: Static-to-dynamic transformation. J. Algorithms 1, 4 (Dec. 1980), 301-358.

5. Blum, N., and Mehlhorn. K. On the average number of rebalancing operations in weight-balanced trees. A-78/06, Fachbereich Angewandte Mathematik und Informatik. Universität des Saarlandes, Saarbrücken. West Germany. 1978.

6. Brown, M.R., and Tarjan. R.E. Design and analysis of data structures for representing sorted lists. SIAM /.Comput. 9, 3 (Aug. 1980), 594-614.

7. Chazelle. B. Filtering search: A new approach to query-answering In Proceedings of 24th Annual IEEE Symposium on Foundations of Computer Science. (Tucson, Ariz., Nov. 7-9, 1983). 122-132.

8. Chazelle. B. How to search in history. Inform. Control, to appear.

9. Chazelle, B., and Guibas, L.J. Fractional cascading: A data structur ing technique with geometric applications. In Automata, Languages, and Programming, 12th Colloquium, (Napflion, Greece, July 15-18. 1985); Lecture Notes in Computer Science 194. Wilfried Bauer, Ed., Springer-Verlag, Berlin, 1985, 90-100.

10. Cole, R. Searching and storing similar lists. I. Algorithms. to appear.

1. Dobkin, D., and Lipton, R.). Multidimensional search problems. SIAM J. Comput. 5. 2 (June 1976). 181-186.

12. Dobkin. D.P., and Munro, J.I. Efficient uses of the past. In Proceedings of 21st Amiual IEEE Symposium on Foundations of Computer Science, (Syracuse. N.Y., Oct. 13-16, 1980), 200-206.

13. Edelsbrunner, H., Guibas, L.J., and Stolfi, J. Optimal point location in a monotone subdivision. Rep. 2, Digital Systems Research Center, Palo Alto, Calif., 1984

14. Guibas, L.J., and Sedgewick, R. $\Lambda$ dichromatic framework for balanced trees. In Proceedings of 19th Annual IEEE Symposium on Foundations of Computer Science, (Ann Arbor, Mich., Oct. 16-18. 1978), $8-21$.

15. Huddleston, S., and Mehlhorn, K. Robust balancing in B-trees, Lecture Notes in Computer Science 104, Springer-Verlag, Berlin, West Germany (1981), 234-244.

16. Huddleston, S., and Mehlhorn, K. A new data structure for representing sorted lists. Acta Inform. 17, 2 (June 1982), 157-184.

17. Huddleston, $S$. An efficient scheme for fast local updates in linear lists. Department of Information and Computer Science, Univ. of California, Irvine, 1981

18. Kirkpatrick, D. Optimal search in planar subdivisions. SIAM J. Comput. 12, 1 (Feh. 198.3), 28-35

19. Knuth, D.E. The Art of Computer Programming Vol. 1: Fundamental Algorithms. 2d. ed. Addison-Wesley. Reading, Mass., 1973.

20. Kosaraju, S.R. Localized search in sorted lists. In Proceedings of 13th Annual ACM Symposium on Theory of Computing, (Milwaukee, Wis., May 11-13, 1981), 62-69.

21. Krijnen, T., and Meertens, L.G.L.T. Making B-trees work for B. IW 219/83. The Mathematical Centre, Amsterdam, The Netherlands, 1983.

22. Lee, D.T., and Preparata, F.P. Location of a point in a planar subdivi sion and its applications. SIAM J. Comput. 6. 3 (Sept. 1977), 594-606

23. Lipton, R.J., and Tarjan, R.E. Applications of a planar separator theorem. In Proceedings of 18th Annual IEEE Symposium on Foundations of Computer Science, (Providence, R.I., Oct. 11-Nov. 2, 1977), 162-170.

24. Lipton, R.J., and Tarjan, R.E. A separator theorem for planar graphs. SIAM J. Appl. Math. 36, 2 (Apr. 1979), 177-189.

25. Maier. D., and Salveter, S.C. Hysterical B-trees. Inform. Process. Lett. 12, 4 (Aug. 13, 1981). 199-202.
26. Mehlhorn, K. Data Structures and Algorithms 1: Sorting and Searching. Springer-Verlag. Berlin, 1984.

27. Myers, E. W. AVL dags. Tech. Rep. 82-9, Department of Computer Science. Univ. of Arizona. Tucson, Ariz., 1982.

28. Myers, E.W. Efficient applicative data types. In Conference Record Eleventh Ammul ACM Symposium on Principles of Programming Languages, (Salt Lake City, Utah. Jan. 15 18, 1984), 66-75.

29. Nievergelt. I., and Reingold, E.M. Binary search trees of bounded balance. SIAM J. Comput. 2, 1 (Mar. 1973), 33-43.

30. Olivié, H. A new class of balanced search trees: Half-balanced binary search trees. RAIRO Informatique Théoretique, 16 (1982), 51-71.

31. Overmars, M.H. Searching in the past I. Inform. Control, to appear.

32. Preparata, F.P. A new approach to planar point location. SIAM J. Comput. 10, 3 (Aug. 1981), 473-482.

33. Reps, T., Teitelbaum, T., and Demers, A. Incremental contextdependent analysis for language-based editors. ACM Trans. Prog. Lang. Syst.. 5 (1983), 449477.

34. Sarnak, N. Persistent data structures. Ph.D. dissertation, Department of Computer Science, New York University, New York, to appear.

35. Sleator. D.D., and Tarjan, R.E. Self-adjusting binary search trees. $A C M$ 32, 3 (July 1985), 652-686.

36. Swart, G. Efficient algorithms for computing geometric intersections Tech. Rep. \#85-01-02, Department of Computer Science, Univ, of Washington, Seattle. 1985.

37. Tarjan, R.E. Data Structures and Network Algorithms. Society for In dustrial and Applied Mathematics. Philadelphia, Pa., 1983.

38. Tarjan, R.E. Updating a balanced scarch trec in $O(1)$ rotations. In form. Process. Lett. 16, 5 (June 1983), 253-257.

39. Tarjan. R.E. Amortized computational complexity. SIAM J. Alg. Disc Meth. 6. 2 (Apr. 1985). 306-318.

40. Tarjan, R.E., and van Wyk. C.J. A linear-time algorithm for triangulating simple polygons. In Proceedings of 18th Annual ACM Symposium on Theory of Computing. (Berkeley. Calif., May 28-30, 1985). to appear.

41. Tsakalidis, A.K. AVL-trees for localized search. Lecture Notes in Computer Science 172, Springer-Verlag, Berlin, West Germany (1984) 473-485.

CR Categories and Subject Descriptors: E.1[Data]: Data Structuresgraphs, lists, trees; E.2[Data]: Data Storage Representations-linked representations; F.2.2[Analysis of Algorithms and Problem Complexity]: Nonnumerical Algorithms and Problems-computations on discrete structures, geometrical problems and computations; I.3.5[Computer Graphics]:

General Terms: Algorithms, Theory

Additional Key Words and Phrases: post-office problem, planar point location, persistent data structure, search tree

Received $8 / 85$; revised $10 / 85$; accepted $2 / 86$

Authors' Present Addresses: Neil Sarnak, IBM T.J. Watson Research Center, P.O. Box 218, Yorktown Heights, NY 10598. Robert E. Tarjan, Computer Science Department, Princeton University, Princeton. NJ 08544, and AT\&T Bell Laboratories, Murray Hill, NI 07974

Permission to copy without fee all or part of this material is granted provided that the copies are not made or distributed for direct commercial advantage, the ACM copyright notice and the title of the publication and its date appear, and notice is given that copying is by permission of the Association for Computing Machinery. To copy otherwise, or to republish, requires a fee and/or specific permission.

\section{CORRIGENDUM}

David S. Scott and S. Sitharama Iyengar, TID-A translation invariant data structure for storing images. Commun. ACM 29, 5(May 1986), 418-429.

Page 425, left column, paragraph 1, sentences 6 and 7 should read:
Maximal square characterization of Figure 9a using TID structure is described in Table II. Table III summarizes the best, worst, and average performance for the various locations. 